\title{
Use of Lung Transplantation Survival Models to Refine Patient Selection in Cystic Fibrosis
}

\author{
Theodore G. Liou, Frederick R. Adler, and David Huang \\ Division of Respiratory, Critical Care, and Occupational Pulmonary Medicine, Department of Internal Medicine, and Departments of Biology \\ and Mathematics, University of Utah, Salt Lake City, Utah; and Neuropsychiatric Institute, University of California, Los Angeles, California
}

\begin{abstract}
Lung transplantation in cystic fibrosis may improve survival for patients with low 5-year predicted survival. Identifying characteristics that affect post-transplantation survival may improve patient selection and survival benefit. Using Cystic Fibrosis Foundation Patient Registry and United Network for Organ Sharing data, we identified 845 lung transplant recipients from 1991-2001, and 12,826 control patients from 1997. We used Cox proportional hazards models to identify variables that influence post-transplantation survival. To estimate the survival benefit of transplantation for patients affected by identified variables, we compared Kaplan-Meier survival curves of transplanted and control patients stratified by 5 -year predicted survival. Post-transplantation survival improved annually. Youth, Burkholderia cepacia, and cystic fibrosis-related arthropathy increased the post-transplantation hazard of death. Compared with control subjects, transplanted adults with a 5-year predicted survival of less than $50 \%$ without $B$. cepacia or arthropathy have improved survival. Transplanted adults with $B$. cepacia, arthropathy, or a 5-year predicted survival of greater than $50 \%$ have decreased survival. Transplantation never improves survivorship for pediatric patients. Patients with arthropathy, B. cepacia infection, or younger age derive no aggregate survival benefit and must appraise carefully the high risk of decreased post-transplantation survival. Adult patients with low 5 -year predicted survival without $B$. cepacia infection should receive priority for lung transplantation.
\end{abstract}

Keywords: age; arthropathy; Burkholderia cepacia; Cox proportional hazards model; organ allocation

Cystic fibrosis (CF) is the most common genetically determined disease in the United States. It causes multisystem disease primarily involving the gastrointestinal tract and the respiratory system. Approximately $80 \%$ of patients die of progressive respiratory disease (1). Lung transplantation, as heart-lung, cadaveric bilateral single lung, and living-donor procedures, remains the most aggressive treatment of end-stage lung disease since its introduction (2-4). However, complications of lung transplantation are the second most frequent cause of death for patients afflicted with $\mathrm{CF}$, accounting for approximately $12 \%$ of deaths in 2002 (1).

Because of the high risks associated with lung transplantation, we recently analyzed its effect on survival in patients with $\mathrm{CF}$ stratified by 5 -year predicted survival. We used the logistic regression survival model of $\mathrm{CF}$ that we previously validated to determine 5-year predicted survival (5). This model includes nine variables and one interaction to make accurate predictions

(Received in original form July 12, 2004; accepted in final form January 22, 2005) Supported in part by the Margolis Family Foundation of Utah and the U.S. Cystic Fibrosis Foundation, Bethesda, Maryland. T.G.L. is a Harry Shwachman Clinical Investigator of the CF Foundation.

Correspondence and requests for reprints should be addressed to Theodore G. Liou, M.D., 26 North Medical Drive, Salt Lake City, UT 84132. E-mail: ted.liou@ utah.edu

Am I Respir Crit Care Med Vol 171. pp 1053-1059, 2005

Originally Published in Press as DOI: 10.1164/rccm.200407-9000C on February 1, 2005

Internet address: www.atsjournals.org of 5-year survival: age, sex, $\mathrm{FEV}_{1}$, weight-for-age $z$ score, pancreatic sufficiency status, diabetes status, Staphylococcus aureus infection status, Burkholderia cepacia infection status, and number of acute exacerbations of $\mathrm{CF}$ in 1 year, and an interaction term between $B$. cepacia and number of acute exacerbations.

Using this model, we demonstrated that only patients with a less than $30 \%$ chance of living 5 years had a clear survival benefit from lung transplantation (6). Lung transplantation decreased survival for patients with a 5-year predicted survival greater than $50 \%$. Patients with a 30 to $50 \%$ chance of 5 -year survival had essentially no survival effect of lung transplantation in 5 years of follow-up. Physicians wishing to apply this model to individual patients can use worksheets that we have provided to manually calculate a 5-year predicted survival (aje.oupjournals.org/cgi/ content/full/153/4/345/DC1) (or contact the authors).

In our earlier analysis, patients stratified before transplantation into different 5-year predicted survivorship groups all had the same post-transplant survivorship $(6,7)$. However, if different factors determine pre- and post-transplant survivorship, patients with similar predicted 5-year nontransplanted survival may have significant differences in post-transplantation survival. Identification of specific pretransplantation variables that predict post-transplantation survivorship may improve patient selection for the procedure.

Patients infected with $B$. cepacia have poor post-transplantation survivorship compared with other transplanted patients $(8,9)$. Nine different $B$. cepacia genomovars appear to alter survivorship to different degrees $(10,11)$. However, because $B$. cepacia markedly reduces nontransplanted survival, there may still be a survival benefit from transplantation despite the reduced post-transplantation survival. There have been no comparisons of survival between transplanted and nontransplanted patients with B. cepacia stratified by expected survival.

Other than B. cepacia, no pretransplantation variables have been shown to affect post-transplantation survival $(12,13)$. We used the U.S. CF Foundation Patient Registry (CFFPR) and the United Network for Organ Sharing (UNOS) database to analyze post-transplantation survivorship to discover additional variables that may help predict success or failure with lung transplantation for patients with end-stage lung disease from CF. The CFFPR contains data for 33,415 unique patients with CF followed at 117 certified CF care centers across the United States (1). An estimated $90 \%$ of all patients with CF in the United States are included in the CFFPR (1). Some of the results of this study have been previously reported in abstract form at the North American CF Conference, 2004, in St. Louis, MO (14).

\section{METHODS}

We used the 1988-2001 CFFPR and the 1988-2002 UNOS data set to identify patients for study. The CF Foundation, UNOS, and the University of Utah Investigational Review Board each reviewed and approved our project. Written consent was waived.

Patients in the transplantation group were matched between the CFFPR and the UNOS data set using exact birth date, sex, and year of transplantation to ascertain exact transplantation dates. We calculated 
survival from the day of transplantation to December 31, 2001, including death or loss to follow-up. Patients were considered lost to follow-up from January 1 of the year after the last recorded data for that patient in the CFFPR

Patients included as controls had not received solid organ transplantation before 1997. To compare transplant survival with the latest available 5-year period for control subjects, survival was calculated from January 1, 1997, to December 31, 2001, death, lung transplantation, or loss to follow-up. Survival was censored on transplantation.

We used our logistic regression model of 5-year survivorship to predict survival for each control and transplant recipient (5). Data required for the calculation included birth date, sex, weight-for-age $z$ score, $\mathrm{FEV}_{1}$ normalized to percent predicted $\left(\mathrm{FEV}_{1} \%\right)$, diabetes status, $S$. aureus and B. cepacia status, and number of acute exacerbations in 1997 for control subjects or in the year before transplantation for transplant recipients (5). We used Splus (Insightful Corp., Seattle, WA) (15) to calculate $\mathrm{FEV}_{1} \%$ and weight-for-age $z$ score and to apply our logistic regression model to calculate 5-year predicted survival, as previously described $(5,16)$.

To detect variables that plausibly alter the hazard of post-transplantation death, we used variables from the CFFPR, excluding those pertaining to clinical investigations or with sparse data. Using forward and backward stepwise selection procedures, we developed a final candidate multivariate Cox proportional hazards model of death after lung transplantation for CF (17).

We created univariate Cox proportional hazards models using each variable. Variables with an absolute $z$ statistic of less than 1 were rejected from further consideration. Remaining variables were candidates for the forward model construction procedure. After creating a multivariate Cox proportional hazards model by the standard forward model construction procedure (17), we applied a backward selection procedure with a criterion of a $\mathrm{p}$ value of less than 0.05 to retain only variables that had a significant effect in the multivariate Cox model (17).

For the backward stepwise selection procedure, we included all variables with sufficient data and plausible biological effect on survival in a multivariate Cox model. We sequentially removed variables with a $p$ value of greater than 0.05 to arrive at a final candidate multivariate Cox model. We examined remaining variables for two-way interactions, eliminating interactions involving small numbers of patients or interactions with year of transplantation because there can be no impact on patient selection (17).

We evaluated the survival effect of variables included in the final multivariate Cox proportional hazards model. For each covariate in the Cox model, we selected a control group of patients and a transplant group of recipients. After selection, we stratified the patients by 5 -year predicted survival of less than $50 \%$, between 50 and $90 \%$, and greater than $90 \%$. Within strata, we used Cox proportional hazards models to test whether transplantation affected survival.

\section{RESULTS}

\section{Patients}

The 1991-2001 CFFPR contains 30,930 patients. From 1991 through 2001,1,379 patients in the CFFPR underwent lung transplantation. We excluded 209 patients who also underwent heart, liver, or other solid organ transplantation, 126 patients missing pulmonary function data, 38 missing microbiology data, and 2 missing weights. Of 1,004 patients for whom we were able to calculate a 5-year predicted survival, we ascertained a transplantation date for 845 , the final number of patients included in this study.

The 1997 cohort of nontransplanted patients numbered 20,650 patients. We excluded 5,604 patients missing pulmonary function information (3,338 were too young for testing, 2,266 were simply missing), 268 patients of ethnic or racial backgrounds for whom we lack equations to normalize $\mathrm{FEV}_{1} \%, 1,133$ patients missing acute exacerbations data, 799 patients missing microbiology data, 13 patients missing pancreatic sufficiency data, and 7 patients missing weight information. This left 12,826 patients for whom we were able to calculate a 5 -year predicted survival.

\section{Model Development}

We started with a total of 24 variables with plausible biological effects for analysis (Table 1). Other than variables included in the 5-year predicted survival model (5), no variables were significant predictors of survival by univariate logistic regression in the control group of patients. The 5-year predicted survival itself was not a predictor of post-transplantation survival using univariate logistic regression. Transplant recipients had lower weight and pulmonary function, and more diabetes, pancreatic insufficiency, infections, and acute exacerbations of disease (Table 2). The incidence of each condition among the transplant recipients was sufficient to test the variables for effects on posttransplantation survival.

Univariate Cox proportional hazards models identified 10 variables as potentially significant predictors of survival with a $p$ value of 0.2 or less: age $(\mathrm{p}=0.0004)$, acute exacerbations $(\mathrm{p}=$ $0.02)$, arthropathy $(\mathrm{p}=0.01)$, B. cepacia $(\mathrm{p}=0.0004), \mathrm{FEV}_{1} \%$ $(\mathrm{p}=0.2)$, liver disease $(\mathrm{p}=0.15)$, mucoid Pseudomonas aeruginosa $(\mathrm{p}=0.065)$, nonmucoid $P$. aeruginosa $(\mathrm{p}=0.18)$, weightfor-age $z$ score $(\mathrm{p}=0.16)$, and year of transplantation $(\mathrm{p}=$ $0.008)$

Forward and backward selection procedures both identified a four-variable candidate model of the hazard of death after lung transplantation for $\mathrm{CF}$, which includes year of transplantation, age at transplantation, infection with $B$. cepacia, and arthropathy (Table 3 ). We additionally considered the effect of $B$. cepacia genomovar but found that there is insufficient information in the CFFPR to analyze the effects after transplantation.

The interaction between age at transplantation and $B$. cepacia was significant and was included in the final Cox proportional hazards model (Table 3$)$. The coefficient for age $(-0.0252$; Table 3 ) shows that the hazard of death decreases with age. The significant interaction with $B$. cepacia infection implies that the age effect on the hazard of death is altered by the status of $B$. cepacia infection. With B. cepacia infection, each additional year of age is associated with a $6 \%$ increase in the hazard of death. Of the nine variables and one interaction in our 5-year predicted survival model of nontransplanted patients with $\mathrm{CF}$, only the

\section{TABLE 1. VARIABLES TESTED FOR SURVIVAL EFFECT}

\section{Age, yr*}

Acute exacerbations, no./yr*

Alcaligenes (Achromobacter) xylosoxidans

Allergic bronchopulmonary aspergillosis

Arthropathy*

Aspergillus species

Atypical mycobacteria

Burkholderia cepacia*

Diabetes status

$\mathrm{FEV}_{1} \%$

Height

Sex, male/female

Liver disease, three variables*

Methicillin-resistant Staphylococcus aureus

Mucoid Pseudomonas aeruginosa*

Nonmucoid Pseudomonas aeruginosa*

Pancreatic sufficiency status

Staphylococcus aureus

Stenotrophomonas maltophilia

Weight-for-age $z$ score*

Year of transplantation*

5-Year predicted survival

* Univariate Cox proportional hazards models found 10 variables were potentially significant predictors of survival ( $p<0.2$; see RESULTS). Stepwise selection procedures eliminate all but age, arthropathy, B. cepacia, and year of transplantation from the multivariate Cox proportional hazards model. 
TABLE 2. PATIENT CHARACTERISTICS AND THE EFFECT OF STRATIFICATION BY 5-YEAR PREDICTED SURVIVAL

\begin{tabular}{|c|c|c|c|c|c|c|}
\hline \multirow[b]{3}{*}{ Variable (except as noted, \% affected) } & \multirow{2}{*}{\multicolumn{2}{|c|}{ All Study Patients }} & \multicolumn{4}{|c|}{ Patients Stratified by 5-Year Predicted Survival } \\
\hline & & & \multicolumn{2}{|c|}{ Control Patients } & \multicolumn{2}{|c|}{ Transplant Recipients } \\
\hline & Control & Transplant & $<50 \%$ & $50-90 \%$ & $<50 \%$ & $50-90 \%$ \\
\hline No. patients & 12,826 & 845 & 579 & 2,539 & 347 & 471 \\
\hline Deaths & 9.3 & 36.0 & 55.4 & 24.1 & 41.8 & 36.1 \\
\hline Age, yr, mean $\pm S D$ & $25.1 \pm 9.1$ & $25.1 \pm 9.0$ & $25.3 \pm 9.1$ & $23.6 \pm 9.7$ & $25.7 \pm 8.2$ & $24.8 \pm 9.6$ \\
\hline Acute exacerbations, mean no./yr \pm SD & $0.90 \pm 1.44$ & $2.75 \pm 1.74$ & $4.05 \pm 1.40$ & $2.15 \pm 1.61$ & $4.02 \pm 1.22$ & $1.93 \pm 1.50$ \\
\hline Arthropathy & 1.42 & 1.78 & 2.9 & 3.2 & 2.6 & 1.3 \\
\hline Burkholderia cepacia & 4.26 & 7.57 & 19.5 & 11.9 & 12.4 & 4.4 \\
\hline Diabetes & 6.55 & 16.6 & 25.7 & 14.4 & 26.8 & 9.5 \\
\hline $\mathrm{FEV}_{1} \%, \% \pm \mathrm{SD}$ & $76.0 \pm 28.8$ & $28.6 \pm 11.8$ & $28.8 \pm 9.4$ & $45.1 \pm 15.1$ & $24.8 \pm 7.3$ & $29.7 \pm 10.6$ \\
\hline Sex, \% male & 47.2 & 47.1 & 52.8 & 50.7 & 53.9 & 43.3 \\
\hline Pancreatic sufficiency & 6.4 & 2.72 & 1.9 & 3.4 & 1.7 & 3.0 \\
\hline Staphylococcus aureus & 42.3 & 23.4 & 20.9 & 30.8 & 18.2 & 25.7 \\
\hline Weight for age $z$ score, \pm SD & $-0.71 \pm 1.05$ & $-1.46 \pm 1.00$ & $-1.96 \pm 0.87$ & $-1.28 \pm 0.95$ & $-1.82 \pm 0.90$ & $-1.22 \pm 0.96$ \\
\hline
\end{tabular}

As a group, transplant recipients have lower $\mathrm{FEV}_{1} \%$ and weight-for-age $z$ score, and more acute exacerbations, pancreatic insufficiency, diabetes, and infections

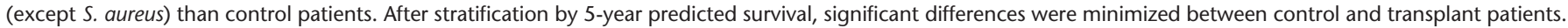

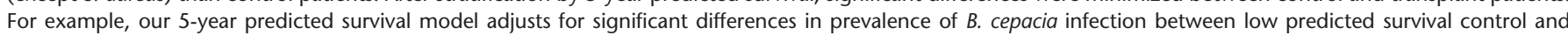
transplant patients.

interaction of age and $B$. cepacia infection status appeared in the final Cox model for post-transplantation survival.

Two variables included in consensus guidelines for patient selection for lung transplantation $(19,20)$, sex and weight, were eliminated from the final Cox model. We examined weight-forage $z$ score distribution among control subjects and transplant recipients to see if there was a selection bias that might have compromised our ability to find a significant influence on posttransplantation survival. We found no evidence of such a bias (Kolmogorov-Smirnov test [18], $\mathrm{p}=0.08$ ).

We illustrate two selected features of our post-transplantation Cox model with Kaplan-Meier survival curves. The progressive improvement in lung transplantation over time is shown by comparing survival for patients transplanted 1991-1997 and 1998-2001 (Figure 1). There were 473 patients who underwent transplantation during 1991-1997 and 372 patients during 1998-2001.

CF-related arthropathy affected 15 patients who underwent lung transplantation. It was not reported in the other 830 patients in our transplant group. Arthropathy had the largest hazard ratio in our Cox model (Table 3). The arthropathy-affected transplant recipients had a much worse post-transplantation survival compared with other transplant recipients (Figure 2). Ten of the patients died during the initial 5 years of follow-up; half of the deaths occurred during the first 6 months post-procedure.

\section{Impact on Patient Selection for Lung Transplantation}

We estimated the survival effect of arthropathy, B. cepacia infection, and age on transplanted patients compared with control subjects stratified by 5-year predicted survival. First, we considered the effect of age on transplant recipients uninfected by $B$. cepacia. Even those children with 5-year predicted survival of less than $50 \%$ had no survival benefit from lung transplantation (Figure 3A). Adults with a less than 50\% 5-year predicted survival had a slight survival disadvantage related to transplantation, which persisted for a year after the procedure, but the survival curves crossed at 1 year and resulted in a net survival benefit of lung transplantation for these patients after 5 years of follow-up (Figure 3C). As expected (6), both children (Figure 3B) and adults (Figure 3D) with 5-year predicted survival between 50 and $90 \%$ had a significant decrease in survival related to lung transplantation.

Second, we considered the effects of age for transplant recipients infected with B. cepacia. Children ( $<18$ years) infected with B. cepacia had no survival effect related to lung transplantation whether they had a 5-year predicted survival of less than $50 \%$ (Figure 4A) or 50 to $90 \%$ (Figure 4B), although it should be noted that there were only nine patients and two deaths in the latter group. Adults with B. cepacia infection with a 5-year predicted survival of less than $50 \%$ (Figure 4C) suffered decreased survival after transplantation. Adults with B. cepacia infection and 5-year predicted survival of 50 to $90 \%$ (Figure 4D) had no survival benefit. Finally, patients with arthropathy and either low or high 5-year predicted survival did poorly with transplantation, primarily because of early postoperative deaths compared with other transplant recipients (Figure 2), and had no

TABLE 3. MULTIVARIATE COX PROPORTIONAL HAZARDS MODEL OF THE HAZARD OF DEATH AFTER LUNG TRANSPLANTATION

\begin{tabular}{lcccc}
\hline Variable & Coefficient & SE & Hazard Ratio & $\begin{array}{c}\text { 95\% Confidence } \\
\text { Interval }\end{array}$ \\
\hline Age & -0.0252 & 0.0067 & 0.975 & $0.96-0.99$ \\
Arthropathy & 0.802 & 0.32 & 2.23 & $1.19-4.18$ \\
B. cepacia & -0.723 & 0.62 & 0.485 & $0.144-1.636$ \\
Year of Transplant & -0.0618 & 0.022 & 0.94 & 0.013 \\
Age $\times$ B. cepacia & 0.0587 & 0.024 & 1.060 & 0.901 \\
\hline
\end{tabular}

The model includes four variables and a single interaction term. B. cepacia as a single variable does not reach statistical significance but is included because of the strong and significant interaction term with age. The model demonstrates that the hazard of death after lung transplantation for cystic fibrosis has improved by approximately 6\%/year between 1991 and 2001 . See text for other discussion and Figure 1. 


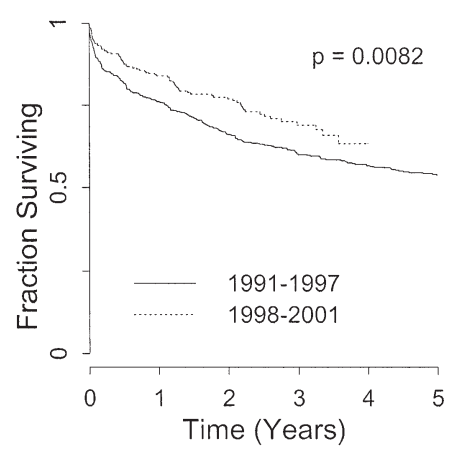

Figure 1. Survival post-transplantation for patients with CF has improved with time as shown by survival in two transplantation eras. Post-transplantation survival for 473 patients (217 deaths) with CF transplantation from 1991 through 1997 is compared to survival for 372 patients ( 87 deaths) transplanted from 1998 through 2001. The hazard ratio of death in each year is $94 \%$ of the hazard in the previous year $(p=0.006$, from Table 3). Thus, for example, the hazard for patients transplanted in 1996 is 0.73 compared with patients transplanted in 1991.

improvement in survival when compared with nontransplanted patients with arthropathy (not shown).

\section{DISCUSSION}

The intention of lung transplantation is to improve outcomes for patients with CF. Clinicians focus on post-transplantation survival as the easiest measure of efficacy. However, post-transplantation survival must be compared with nontransplanted survival to obtain a complete picture of the survival effect of transplantation. Factors present pretransplantation that affect post-transplantation survival may help identify individuals who will have improved posttransplantation survivorship.

A transplantation selection factor, such as age, that predicts longer post-transplantation survival is an indication for transplantation only if it also predicts an increase in survival compared with nontransplantation. A factor that predicts decreased survival in a transplant recipient relative to other transplant recipients might still result in survival benefit when comparing post-transplantation survival to nontransplanted survival within a 5-year predicted survival stratum. In the case of patients with $B$. cepacia, infection decreases survival post-transplantation but also decreases nontransplanted survival. Potentially, the negative survival impact of $B$. cepacia infection might be lessened in transplanted patients compared with nontransplanted control subjects, resulting in a survival benefit from the procedure.

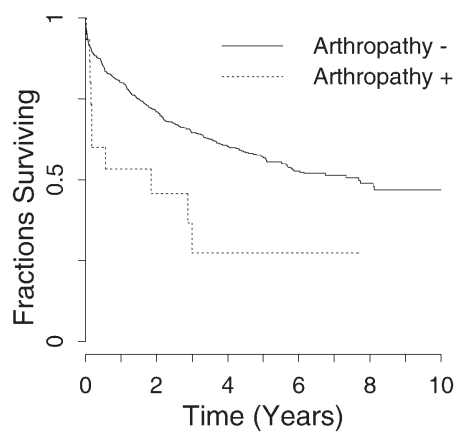

Figure 2. Substantial effect of arthropathy on post-transplantation survival is shown. Patients with $\mathrm{CF}$ and with arthropathy were rare as reflected in the small number of transplant recipients with arthropathy (15). Survival was compared with 830 transplant recipients not affected by arthropathy. Despite small numbers, the effect of arthropathy on post-transplantation survival was profound (hazard ratio, 2.23; $p=0.013$; from Table 3) and persisted beyond 5 years of follow-up. There were 10 deaths among the 15 transplant recipients with arthropathy compared with 320 deaths among the 830 recipients without arthropathy.

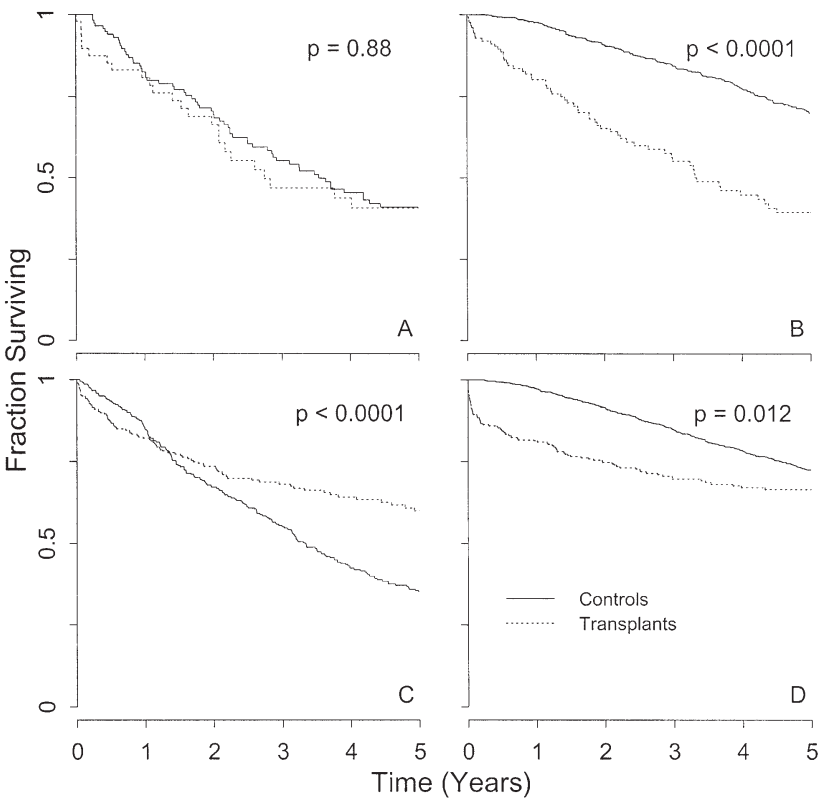

Figure 3. Age affects survival following lung transplantation for CF. Patients infected with B. cepacia are excluded from this analysis. Year of transplantation had no correlation with either age at transplantation or 5-year predicted survival. Patients with arthropathy are included in this analysis but were too few to have any effect on results. $(A)$ Children with a 5 -year predicted survival of less than $50 \%$ had no survival benefit from lung transplantation. Survival for 57 transplant recipients younger than 18 years ( 28 deaths) and 139 control subjects ( 73 deaths) is shown. (B) Children with a 5-year predicted survival between 50 and $90 \%$ had decreased survivorship from lung transplantation. Survival for 127 transplant recipients ( 61 deaths) and 720 control patients ( 181 deaths) is shown. (C) Adults with a 5-year predicted survival of less than $50 \%$ had a survival advantage from transplantation. Survival for 362 control patients (198 deaths) and 258 transplant recipients (with 85 deaths) is shown. $(D)$ Adults with a 5 -year predicted survival between 50 and $90 \%$ did poorly with lung transplantation. Survival for 330 transplant recipients (96 deaths) and 1,532 control patients (354 deaths) is shown.

We examined 24 clinical variables, including 5-year predicted survival (Table 1), to discover whether any had a significant effect on survival after lung transplantation. The 5-year predicted survival itself and variables incorporated into the 5-year predicted survival prediction other than age or $B$. cepacia infection had no effect on post-transplantation survival (Table 3) $(6,7)$.

Body weight is used by $79 \%$ of lung transplantation centers in the United States to select patients for the procedure (21). Our analysis found that there was no effect of body weight or of other nutritional factors on post-transplantation survival (Table 1). Patient selection procedures that use body weight already in place at transplant centers might have biased our analysis, but we found that there was no difference in weightfor-age $z$ score distribution between transplanted and nontransplanted patients with 5-year predicted survival less than 50\%. This suggests that body weight may not be rigorously used as a selection criterion for transplantation, but our analysis suggests that there may be no need for this criterion.

A sex-based selection preference for lung transplantation has been suggested $(19,20)$ because of the decreased survival of females with CF compared with males $(5,22)$. Our method of patient selection for transplantation (6) incorporates sex into the calculations of weight-for-age $z$ score, $\mathrm{FEV}_{1} \%$, and 5 -year predicted survival. Our new finding that sex caused no direct 


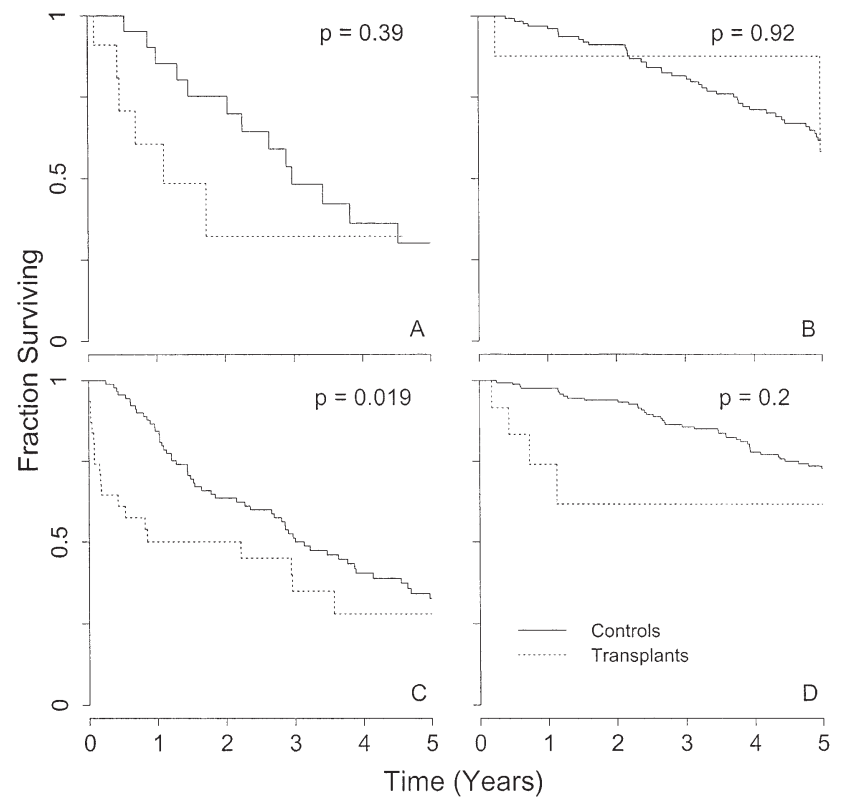

Figure 4. The survival effect of $B$. cepacia infection for lung transplant recipients by age and predicted survival status is shown. For children infected with $B$. cepacia, survival is unchanged by lung transplantation whether 5-year predicted survival is $(A)$ less than $50 \%$ or $(B)$ between 50 and $90 \%$. A shows survival of 22 control patients younger than 18 years (13 deaths) and 12 transplant recipients younger than 18 years (6 deaths). B shows survival of 127 control patients younger than 18 years (42 deaths) and 9 transplant patients younger than 18 years (2 deaths). (C) Adults with $B$. cepacia infection suffered a survival decrease with lung transplantation even though nontransplanted 5-year predicted survival was already poor. Survival of 91 control patients (55 deaths) and 31 transplant recipients (19 deaths) is shown. (D) Adults with $B$. cepacia infection with a pretransplantation 5 -year predicted survival between 50 and $90 \%$ had no survival benefit with lung transplantation. Survival of 174 control patients ( 42 deaths) and 12 transplant recipients (4 deaths) is shown.

post-transplantation survival effect demonstrates that it need not be separately considered as a criterion for lung transplantation.

Somewhat unexpectedly, we found a significant reduction in hazard of death post-transplantation for patients who underwent transplantation later during the study period (Table 3). Careful inspection of the Kaplan-Meier survival plot comparing the 19982001 cohort of transplanted patients with the 1991-1997 cohort revealed that the gain appears to be because of improved survival during the first 6 months post-transplantation (Figure 1).

Three patient-specific factors available before transplantation appear to significantly alter post-transplantation survival: $\mathrm{CF}$ related arthropathy, B. cepacia infection, and younger age. $\mathrm{CF}$ related arthropathy is a variable, ill-defined condition with no clear etiology, diagnostic algorithm, or uniformly effective treatment (23). Patients frequently have joint pain but lack history of injury, clear association with other characteristic findings of $\mathrm{CF}$, evidence of articular erosions or positive markers, such as rheumatoid factor, or autoimmune-related arthritis. In our prior study of survival in CF (5), arthropathy had no impact on survival. However, in recipients of lung transplantation, arthropathy appeared to have a profound independent negative effect on post-transplantation survival, especially within 2 months of transplantation (Figure 2).

Our results, although significant (Table 3), depend on the outcomes of only 15 transplanted patients with CF-related ar- thropathy compared with 830 transplant recipients without arthropathy. Arthropathy is likely to be greatly underreported. Up to one-third of patients with CF have some type of arthropathy or arthritis (23). However, only $1.5 \%$ of patients in our control group were recorded to have arthropathy. These results suggest that clinicians should take arthropathy into account when considering lung transplantation. But arthropathy should not be considered a contraindication until there is a better disease definition, better reporting, more information about pathophysiology, and more long-term post-transplantation survivorship data.

The hazard of death decreased with increasing age at transplantation. One quarter of transplanted patients were younger than 18 years. Comparison of survival between young transplanted and control patients without $B$. cepacia infection showed that younger patients did not derive survival benefit from lung transplantation, even with low 5-year predicted survival (Figure 3A). The survival benefit of lung transplantation among patients with low 5-year predicted survival seen in our earlier study came entirely from adult transplant recipients (Figure 3C) (6).

Using CFFPR and UNOS data, we can only speculate about possible causes for the increased hazard of death after transplantation in pediatric patients with $\mathrm{CF}$. We found a nonsignificantly higher hazard of death among patients aged 14 to 17 years compared with even younger transplant recipients (data not shown). Rapid growth, physical and emotional maturation, and large social changes occur in this age range. These factors may contribute to poorer transplantation outcomes. For example, young transplant recipients likely benefit from constant parental reminders to take transplantation medications but, as a consequence, may not internalize understanding of the need for treatment. This may predispose to decreased compliance with post-transplantation management in adolescence, a period when conflicts between children and parents often arise in the $\mathrm{CF}$ as well as in the general population.

Our results may suggest a rigid cutoff age of 18 for lung transplantation. Comparison between pediatric recipients and the next quartile of transplant recipients, aged 18 to 24 years, showed a sharp and significant improvement in survival benefit when transplanted survival was compared with nontransplanted survival (data not shown). Young patients, their families, and physicians contemplating lung transplantation must consider that increased survival is relatively unlikely, especially if the patient is younger than 18 years with a 5-year predicted survival greater than $50 \%$.

Immediate or rapid transplantation in the pediatric age group should be considered only if there are compelling reasons for transplantation other than improved survival. Those pediatric patients weighing the risks and benefits of transplantation during adolescence may wish to consider maximizing other therapies for $\mathrm{CF}$ and delaying transplantation until after reaching age 18. Besides moving into a more favorable transplant group (Figure 3 ), the passage of time may allow significant improvements in the procedure itself (Figure 1).

Our Cox model confirms past reports of decreased posttransplantation survival for patients infected with $B$. cepacia compared with post-transplantation survival of patients without B. cepacia infection $(8,10,11)$. Our sample of $B$. cepacia-infected transplant recipients is larger than any prior report, allowing our model to detect a strong interaction between B. cepacia infection and age (Table 3 ) and allowing us to analyze survival effect stratified by age and 5-year predicted survival.

Because of the interaction, pediatric transplant recipients with B. cepacia infection did not suffer decreased post-transplantation survival relative to uninfected pediatric recipients of transplantation. In contrast, adult transplant recipients had a marked decrease in survival related to B. cepacia infection. Comparison of post- 
transplantation survival to nontransplanted survival in patients stratified by 5 -year predicted survival showed no survival benefit of lung transplantation regardless of 5-year predicted survival for young patients (Figures 4A and 4B) and adult patients with high predicted survival (Figure 4D), and actual harm for adult patients with low predicted survival (Figure 4C).

Our study has some limitations. It is retrospective, and selection of patients for lung transplantation may have changed during the period of study. We have limited ability to detect biases in selection for transplantation, and the use of our 5-year survival prediction model to stratify patients for analysis may not have fully controlled for subtle biases. Using the 5-year predicted survival excluded a few patients who were too ill to perform pulmonary function testing. However, we were able to include $84 \%$ of transplanted patients and $87 \%$ of control patients older than 5.5 years. Although our results apply only to patients with complete data for calculating a 5-year predicted survival, the high percentage of inclusion suggests broad generalizability.

We could not consider other variables, not tracked by the CFFPR, that may have a significant impact on survival in $\mathrm{CF}$ and lung transplantation. For example, our model did not consider hypercapnia, which may independently identify gravely ill patients (24). Patients so desperately ill as to require mechanical ventilation have been shown to have survival benefit from lung transplantation if suitable organs could be found in time (25, 26). In practice, however, the challenge of finding suitable organs in time for such patients is often insurmountable.

Furthermore, this retrospective study cannot address all questions about the survival effect of $B$. cepacia on lung transplantation. We cannot account for the possibility of improving treatments for B. cepacia infection with and without transplantation that might have improved outcomes (8). Because there is insufficient information in the CFFPR, we were unable to distinguish differing survival patterns among patients infected with different genomovars of B. cepacia. Although patients with B. cepacia infection did poorly with transplantation and did not have a survival benefit from the procedure, we cannot tell if there might be a subset of patients with $B$. cepacia who would do better. Although collection of additional genomovar information is desirable, we are uncertain if it will be helpful. New information suggests that infections even by a single genomovar of $B$. cepacia may have greatly differing severity depending on the patient or geographic region (27).

Finally, our study, by the nature of the data, cannot give direct guidance to those patients seeking improved quality of life even at the expense of shortened survival. As we have previously conjectured, poor quality of life may correlate with low 5-year predicted survival $(6,7,28)$. Thus, the greatest improvement in quality of life may accompany the greatest survival benefit of lung transplantation. Data do not exist that would allow a test of this hypothesis. We are hopeful, however, that new methods for measuring quality of life will facilitate such a test (29).

Clinicians should seek lung transplantation for patients only if there is an increase in survival relative to nontransplanted survival. They should avoid transplantation for patients who will suffer harm, even if post-transplantation survival appears favorable compared with other transplant recipients. Focusing only on post-transplantation survival without considering survival benefit relative to nontransplantation risks maximizing graft survival at the expense of decreased patient survival. For patients likely to have survival benefit, the amount of benefit and the length of post-transplantation survival may help decide how best to allocate scarce donor organs.

Our validated 5-year predictive model made it possible to stratify transplanted and control patients by survivorship. Our study confirms that patients with 5-year predicted survival greater than $50 \%$ should not be transplanted in order to avoid reducing survival. Moreover, we found no survival benefit of lung transplantation for children or for patients of any age infected with $B$. cepacia. For patients with 5-year predicted survival of less than $50 \%$, we recommend that patients and physicians temper their expectations of survival benefit from lung transplantation when patients are young or infected with B. cepacia. CF-related arthropathy should be a concern for possible transplant recipients but not a contraindication. Because there is an ongoing shortage of suitable organs for transplantation, those patients seeking improved quality of life despite a likely reduction in survival should be deferred in favor of patients likely to have survival benefit from transplantation in addition to any possible improvement in quality of life because of better lung function.

Conflict of Interest Statement: T.G.L. does not have a financial relationship with a commercial entity that has an interest in the subject of this manuscript; F.R.A. does not have a financial relationship with a commercial entity that has an interest in the subject of this manuscript; D.H. does not have a financial relationship with a commercial entity that has an interest in the subject of this manuscript.

Acknowledgment: The authors thank the CF Foundation and UNOS for providing access to data. They thank Barbara C. Cahill, M.D., for thoughtful suggestions, and Donald Alexander, Pharm.D., for helpful suggestions and specific new citations. We thank Maureen McBride and Yulin Cheng at UNOS and Bruce Marshall and Ase Sewall at the CF Foundation for their assistance in obtaining data used in this study.

\section{References}

1. Cystic Fibrosis Foundation. Cystic Fibrosis Foundation Patient Registry 2002 annual data report to the center directors. Bethesda, Md: Cystic Fibrosis Foundation; 2002.

2. Scott J, Higenbottam T, Hutter J, Hodson M, Stewart S, Penketh A, Wallwork J. Heart-lung transplantation for cystic fibrosis. Lancet 1988;2:192-196.

3. Ramirez JC, Patterson GA, Winton TL, de Hoyos AL, Miller JD, Maurer JR. Toronto Lung Transplant Group. Bilateral lung transplantation for cystic fibrosis. J Thorac Cardiovasc Surg 1992;103:287-294.

4. Starnes VA, Barr ML, Cohen RG, Hagen JA, Wells WJ, Horn MV, Schenkel FA. Living-donor lobar lung transplantation experience: intermediate results. J Thorac Cardiovasc Surg 1996;112:1284-1291.

5. Liou TG, Adler FR, Fitzsimmons SC, Cahill BC, Hibbs JR, Marshall BC. Predictive five year survivorship model of cystic fibrosis. Am J Epidemiol 2001;153:345-352.

6. Liou TG, Adler FR, Cahill BC, Fitzsimmons SC, Huang D, Hibbs JR, Marshall BC. Survival effect of lung transplantation for patients with cystic fibrosis. JAMA 2001;286:2686-2689.

7. Liou TG, Adler FR, Cahill BC, Marshall BC. Priorities for lung transplantation among patients with cystic fibrosis. JAMA 2002;287:1525.

8. Chaparro C, Maurer J, Gutierrez C, Krajden M, Chan C, Winton T, Keshavjee S, Scavuzzo M, Tullis E, Hutcheon M, et al. Infection with Burkholderia cepacia in cystic fibrosis: outcome following lung transplantation. Am J Respir Crit Care Med 2001;163:43-48.

9. Egan TM, Detterbeck FC, Mill MR, Bleiweis MS, Aris R, Paradowski L, Retsch-Bogart G, Mueller BS. Long term results of lung transplantation for cystic fibrosis. Eur J Cardiothorac Surg 2002;22:602-609.

10. Aris RM, Routh JC. LiPuma JJ, Heath DG, Gilligan PH. Lung transplantation for cystic fibrosis patients with Burkholderia cepacia complex: survival linked to genomovar type. Am J Respir Crit Care Med 2001;164:2102-2106.

11. De Soyza A, McDowell A, Archer L, Dark JH, Elborn SJ, Mahenthiralingam E, Gould K, Corris PA. Burkholderia cepacia complex genomovars and pulmonary transplantation outcomes in patients with cystic fibrosis. Lancet 2001;358:1780-1781.

12. Aurora P, Gassas A, Ehtisham S, Whitehead B, Whitmore P, Rees PG, Tsang VT, Elliott MJ, De Leval M. The effect of pre-lung transplant clinical status on post-transplant survival of children with cystic fibrosis. Eur Respir J 2000;16:1061-1064.

13. Trulock EP, Ardehali A, Garrity ER Jr, Grover FL, Robbins MK. OPTN/ UNOS Conference on Lung Allocation Policy; March 13, 2003; Chicago, IL.

14. Liou TG, Adler FR, Huang D. Survival after lung transplantation for cystic fibrosis: implications for patient selection. Pediatr Pulmonol 2004;38:319.

15. Chambers JM, editor. Programming with data: a guide to the S language. New York: Springer; 1998. 
16. Hankinson JL, Odencrantz JR, Fedan KB. Spirometric reference values from a sample of the general US population. Am J Respir Crit Care Med 1999;159:179-187.

17. Klein JP, Moeschberger ML. Semiparametric proportional hazards regression with fixed covariates. In: Klein JP, Moeschberger ML, editors. Survival analysis: techniques for censored and truncated data, 1st ed. New York: Springer; 1997. p. 229.

18. Zar JH. Biostatistical analysis, 4th ed. Upper Saddle River, NJ: Prentice Hall; 1998.

19. Yankaskas JR, Mallory GB Jr. The Consensus Committee. Lung transplantation in cystic fibrosis. Chest 1998;113:217-226.

20. Maurer JR, Frost AE, Glanville AR, Estenne M, Higenbottam T, Aris R, Barbers RG, Barst R, Baz MA, de Boer W, et al. International guidelines for the selection of lung transplant candidates. Am J Respir Crit Care Med 1998;158:335-339.

21. Levine SM. The Transplant/Immunology Network of the American College of Chest Physicians: a survey of clinical practice of lung transplantation in North America. Chest 2004;125:1224-1238.

22. Rosenfeld M, Davis R, FitzSimmons S, Pepe M, Ramsey B. Gender gap in cystic fibrosis mortality. Am J Epidemiol 1997;145:794-803.

23. Johnson S, Knox AJ. Arthropathy in cystic fibrosis. Respir Med 1994; 88:567-570.
24. Kerem E, Reisman J, Corey M, Canny GJ, Levison H. Prediction of mortality in patients with cystic fibrosis. N Engl J Med 1992;326:11871191.

25. Massard G, Shennib H, Metras D, Camboulives J, Viard L, Mulder DS, Tchervenkov CI, Morin J-F, Giudicelli R, Noirclerc M. Double-lung transplantation in mechanically ventilated patients with cystic fibrosis Ann Thorac Surg 1993;55:1087-1092.

26. Sood N, Paradowski LJ, Yankaskas JR. Outcomes of intensive care unit care in adults with cystic fibrosis. Am J Respir Crit Care Med 2001; 163:335-338.

27. Manno G, Dalmastri C, Tabacchioni S, Vandamme P, Lorini R, Minicucci L, Romano L, Giannattasio A, Chiarini L, Bevivino A. Epidemiology and clinical course of Burkholderia cepacia complex infections, particularly those caused by different Burkholderia cenocepacia strains, among patients attending an Italian cystic fibrosis center. J Clin Microbiol 2004:42:1491-1497.

28. Liou TG, Cahill BC, Adler FR, Marshall BC. Selection of patients with cystic fibrosis for lung transplantation. Curr Opin Pulm Med 2002;8: 535-541.

29. Quittner AL, Sweeny S, Watrous M, Munzenberger P, Bearss K, Nitza AG, Fisher LA, Henry B. Translation and linguistic validation of a disease-specific quality of life measure for cystic fibrosis. $J$ Pediatr Psychol 2000;25:403-414. 